\title{
The design and implementation of one type of radar signal analyzer
}

\section{Zhang Yongzhen,Zhang Xuezhi,Hou Fei,Shao Chunyu}

\author{
Wuhan Mechanical Technology Colledge, Wuhan, China
}

Keyword: radar、signal collection、bluetooth、database、Fault diagnosis

\begin{abstract}
The design of radar signal analysis was presented in this paper, adopt the method of cable collection, wireless transmission, realize the radar signal test online. established the database of test nodes, board card, Subsystem combination, the fault relationship, structure, circuit diagram, maintenance resources such as database, fulfill the dynamic link of database, analyze node connection relationship, signal feature(waveform 、 frequency 、 circuit 、 structure location) by contrasting data to form Nonlinear fault diagnosis to eliminate radar fault.

One type of radar integrate laser, TV, infrared, the fire control computer, and X/Ka band radar, it is complete function, advanced technology, complicated system and hard technical support. Signal analyzer can test radar signal parameters on line, provide a kind of effective detection methods for equipment maintenance.
\end{abstract}

\section{The system general design}

\subsection{System composition}

Signal analyzer consist of two parts of software and hardware, hardware consist of five part of radar signal adapter, signal collection equipment, wireless communication, signal processing module and signal analyzer host, software consist of database, data analysis software, system control software. The principle diagram as shown in figure 1.

\subsection{System function}

\subsubsection{Real-time detection of radar signal on line}

The signal parameters can be detected in working state by adaptor.

There are two ways to collect the radar signal. The first method detect Dc signal、 power frequency signal by handy wireless collector, realize the data communication between collector and analyzer host by bluetooth; The second method detect the signal by signal acquisition card input interface of analyzer host when the signal is transmitted in high speed in cable.

\subsubsection{Analysis of characteristics of the tested signal}

Analyzer host store 、 process 、 extract signal parameters of collected signal, dynamic link with database of test not 、 board card 、subsystem combination 、 fault relationship 、structure 、 circuit diagram、 maintenance resources, contrasted analyze, display node connection relationship between up and down、 signal characteristics (waveform, frequency, circuit, location), form a scientific nonlinear fault diagnosis, eliminate equipment failure.

\section{System implementation}

\subsection{The design and implementation of testing radar signal online}

\subsubsection{Radar signal adapters}

It should analyze and classify to the joint in optimization, general principle when design adapter. The adaptor is designed to square with detection holes Up and down to facilitate measurement.

The device collect the I/O signal of subsystem in cable. Put the corresponding adapter between 
the I/O Cable end and subsystem socket when testing, can test and maintenance I/O signal of subsystem when radar is on working state.

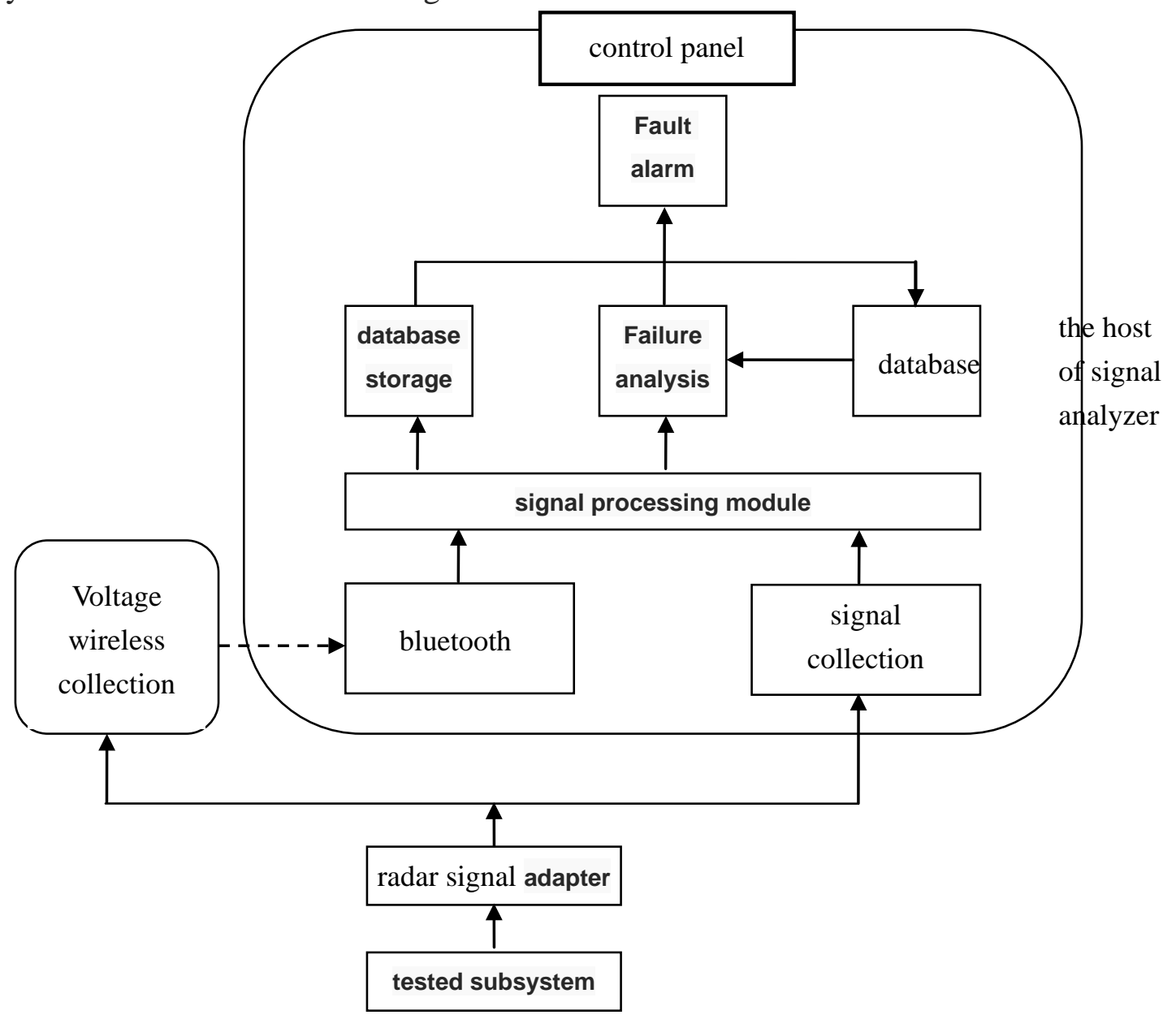

\subsection{2 testing of radar signal}

Figure 1 The principle diagram of signal analyzer

Signal analyzer design data acquisition and processing system of radar signal, mainly includes of signal conditioning circuit, A/D converter circuit, FPGA circuit, USB circuit and power supply module.

\subsubsection{1 signal preprocessing circuit}

signal preprocessing circuit using the ADT1-1 produced by Mini-Circuits to preprocess the analog signal, made the voltage range of input signal can satisfy the demands of A/D converter input, and fulfill the transformation function of single-ended or differential input form.

\subsubsection{Converter circuit}

Converter circuit using the AD9238 produced by ADI company to convert analog signals into digital signals. The chip has 20/40/65 MSPS several different sampling rate, 40MSPS was be selected. And the chip is a dual channel sampling device, a resolution of 12 bit, a large dynamic range, the analog input range: $1 \mathrm{Vp}-\mathrm{p}$ to $2 \mathrm{Vp}-\mathrm{p}$.

\subsubsection{FPGA circuit}

FPGA is the abbreviation of field programmable gate array. Main fulfill the function of sampling data storage, data processing, programming according to different requirement of data processing, has the characteristics of the programmable.

\subsubsection{USB circuit}

USB circuit communicate between FPGA and PC.

\subsubsection{Power supply module}


This power source adopts the $+5 \mathrm{v}$ power supply, and FPGA needs $+3.3 \mathrm{~V} 、+1.8 \mathrm{~V} 、+2.5 \mathrm{~V}$ several kind of voltage, AD9238 needs +3.3V, so this system using LT1764-3.3、LT1764-1.8 and LT1764AEQ several kind of power supply module. supply voltage of LT1764AEQ can be adjusted by Changing peripheral resistance partial pressure. Each power supply module can provide power supply current of $1 \mathrm{~A}$ to meet the request of data acquisition and processing system.

\subsection{Design and implementation of Bluetooth interface circuit}

Bluetooth interface circuit using the Integrated micro controller CY7C68013 of USB2.0 of Cypress company, the chip has enhanced 8051 core, the standard can be compatible with 8051.The chip integrated with a smart USB serial interface engine, two USB data transceiver, a 16-bit address lines, 3 eight bite I/O port, 4K BFIFO.

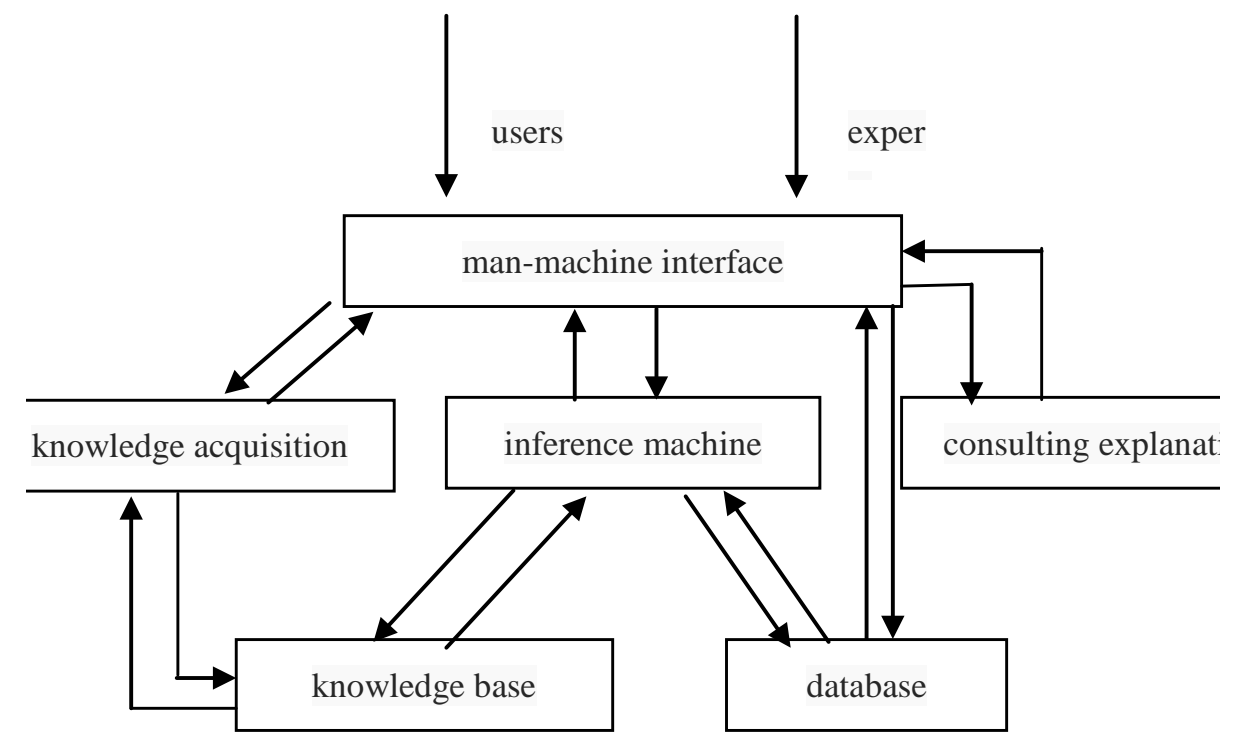

Figure 2 system software composition

\section{3 system software}

Analyzer software consist of database, data analysis software, system software, etc, software composition block diagram as shown in figure 2.

\subsection{1 database}

database use Lab view programming to design combine with SQL. System create databases of radar principle circuit diagram, structure, test nodes, circuit boards, relationship between the database and parameter, each database realize dynamic correlation, fulfill the comparison and analysis of tested signal and fault diagnosis.

The creation of the database mainly lies in the secondary processing of knowledge material, confirm the level of the knowledge description through classification, sorting, set up a corresponding file data tables respectively for call and inquiry. Mainly includes: data table structure design, the design that file perform in a table, image processing, the location of measuring point, etc.

\subsubsection{Data analysis software}

Database using M: N multidimensional data tables correlation method, the complex fault logic stores in a tree structure. Using the SQL language to dynamic inquire the signal node table, electrical logic table, fault logic table, signal characteristic table. Data analysis software processes the signal obtained by terminal, extract the signal parameters, make the fault diagnosis analysis method just like real operation.

\subsection{The design and implementation of fault diagnosis based on dynamic data link}

Signal analyzer use the Lab VIEW virtual instrument technology, MySQL database 
technology, $\mathrm{C}++$ underlying system control technology, Flash multimedia management technology and the Action Script/Java scripting language, build inspection maintenance training platform with database and dynamic operation as the core. And the hardware system has extended interface, can realize data acquisition, analysis and troubleshooting combine with professional testing tools.

Fault diagnosis system consist of data query module, simulation diagnosis module, system management module, tool module, help module, realize different function under the unified scheduling by system.

The characteristics of integrated development platform, interactive programming, the rich panel function and library functions, more powerful, more flexible in application and can be run under multiple operating systems.

The simulation diagnostic modules provide users with the simulation diagnostic model. users can offline learn or familiar with electrical fault diagnosis process, user can complete conventional electrical fault diagnosis simulation without real equipment and hardware interface card; user can complete electrical fault diagnosis training and simulation using the virtual instrument for testing according to the system diagnostic hint.

\section{The characteristics of system}

\subsection{A variety of means to collect radar signal real-time}

According to different features radar signal of all extension test nodes, respectively uses the method of cable high-speed data acquisition and wireless remote acquisition to improved the convenience of radar signal test and analysis.

\subsection{Analyzing the collected signal feature to realize nonlinear fault diagnosis by dynamic linking database.}

Signal analyzer analyze the feature of signal parameters feature, dynamic link with databases of test nodes, the board, extension combination, the relationship between the fault, structure, circuit diagram, display the node structure, electrical and fault logic relationship, form a scientific logic judgment of troubleshooting.

\section{Conclusion}

Signal analyzer has the function of real-time detection of radar signal, signal analysis and fault diagnosis, can fulfill online test of radar signal by using wired and wireless method; query dynamic linking database through kinds of equipment electrical structure, physical structure, logical structure and fault structure to achieve rapid, scientific, dynamic diagnosis equipment failure.

\section{References}

[1]Li Xiao. Indoor layout and configuration [M]. Background of science and technology news, 2000 [2]Lai fengwen. Interior design principle [M]. China building industry press. 2006

[3]Li Lairong. Indoor foliage and decoration [M]. China forestry publishing house

[4]Li qing. Modern afforest adornment of the bedroom [J]. China's health issue. 1996

[5]Chen Song. 100 kinds of healthy indoor plants [M]. Harbin publishing house. 2008 